\title{
marges Marges
}

revue d'art contemporain Revue d'art contemporain

\section{2 | 2021}

\section{La circulation des idées dans l'art contemporain}

\section{Éditorial}

Jérôme Glicenstein

\section{(2) OpenEdition}

\section{Journals}

Édition électronique

URL : https://journals.openedition.org/marges/2411

DOI : 10.4000/marges.2411

ISSN : 2416-8742

\section{Éditeur}

Presses universitaires de Vincennes

\section{Édition imprimée}

Date de publication : 20 mai 2021

Pagination : 5-8

ISBN : 978-2-37924-150-5

ISSN : 1767-7114

\section{Référence électronique}

Jérôme Glicenstein, «Éditorial », Marges [En ligne], 32 | 2021, mis en ligne le 20 mai 2021, consulté le 04 juin 2021. URL : http://journals.openedition.org/marges/2411 ; DOl : https://doi.org/10.4000/ marges. 2411 


\section{Éditorial}

Les années 1960-1970 ont vu se multiplier les références théoriques en art, en lien avec d'importantes transformations sociales et institutionnelles: élévation du niveau de formation des artistes, apparition d'une critique d'art plus universitaire et théoricienne, émergence de la nouvelle figure du curateur comme concepteur d'expositions, politisation du champ artistique, etc. Si cette période très "théoriciste " semble s'être refermée, la place des idées dans l'art contemporain est pourtant loin d'avoir décru. Au contraire, les catalogues et les revues spécialisées, les déclarations d'intention des curateurs ou des artistes foisonnent de références à des intellectuels (Agamben, Butler, Deleuze, Foucault, Haraway, Latour, Rancière, etc.) et à des concepts en vogue - de l'anthropocène à l'object-oriented ontology, en passant par les propositions d'esthétiques accélérationniste, décoloniale ou post-internet, pour citer pêle-mêle quelques exemples récents. Réciproquement, philosophes, scientifiques et essayistes sont régulièrement invités à collaborer à des expositions, des formations, des workshops, etc.

Le champ de l'art contemporain connaît donc une intense circulation d'idées, dont les provenances comme les finalités sont nombreuses et variées. Ce phénomène est parfois regardé avec une certaine condescendance par les universitaires, du fait des confusions et des effets de mode qui tendent à accompagner l'introduction, parfois superficielle ou 
ostentatoire, de nouvelles idées dans le monde de l'art contemporain. Celles-ci méritent pourtant d'être prises au sérieux et examinées, tant pour leur intérêt théorique que dans leurs usages pratiques. Quelles sont leurs origines et comment se diffusent-elles? Quels rôles jouent-elles dans les activités, les représentations, les stratégies de légitimation des artistes, des professionnels et des institutions du monde de l'art? Ces questions permettent d'engager une réflexion sur la place, à la fois marginale et hautement valorisée, qu'occupe le champ artistique dans l'économie générale du champ intellectuel. L'art contemporain apparaît en effet aujourd'hui comme un lieu propice à la construction de certaines carrières intellectuelles, selon d'autres modalités que celles du champ universitaire ou médiatique. Certaines notoriétés s'y affirment, certaines idées nouvelles y émergent, et peuvent être ensuite exportées vers d'autres champs. Les mondes de l'art sont-ils donc susceptibles, à partir de ces " braconnages " théoriques, de devenir à leur tour des foyers de production et de diffusion d'idées au-delà de leurs frontières?

Les premiers textes du dossier ont une approche plus distanciée historiquement. Marie Vicet revient sur "Les Immatériaux », l'exposition organisée autrefois par Jean-François Lyotard au Centre Pompidou. À l'époque, la question n'était pas tant de légitimer la création contemporaine en faisant appel à un philosophe réputé que de donner à voir des idées par le biais d'une exposition. Les artistes contemporains n'étaient d'ailleurs pas particulièrement présents, pas plus que les explications sur les enjeux du projet. Si cette exposition témoigne de changements techniques et sociétaux dont nous sommes les héritiers, il n'en demeure pas moins que cet ensemble ambitieux est resté largement hors de portée de ses destinataires, lesquels étaient privés de toute explication au cours de leur visite - cette mauvaise transmission du message étant d'ailleurs incluse dans le projet même de l'exposition.

Juliette Pym s'intéresse quant à elle aux prémices de l'utilisation par les artistes de références théoriques, à travers l'exemple de l'Independent Study Program du Whitney Museum. Ce programme, décrit comme le lieu du passage entre la théorie critique héritée de l’École de Francfort et la conception de pratiques artistiques interdisciplinaires, tournées 
vers le questionnement de leurs propres conditions est né à la suite des expérimentations néo-avant-gardistes new-yorkaises des années 1960 et a constamment été nourri des réflexions produites par la revue October. À travers l'utilisation de ces modèles, renforcés par une pédagogie radicale, l'idée d'une éthique de la pratique artistique y a été développée comme la condition d'une mise en pratique méthodique et nécessaire de la critique, dans les mondes de l'art et au-delà.

La deuxième partie du numéro concerne des situations plus récentes. Philippe Le Guern s'interroge sur l'influence de l'anthropologie sur certains courants récents de l'art contemporain. Un constat est fait: "le tournant ethnographique de l'art se caractérise par une politisation de l'esthétique tandis que le tournant artistique de l'ethnographie se caractériserait par une esthétisation de la pensée anthropologique ». Les artistes dont les objectifs sont d'intervenir dans des situations sociales font-ils pour autant œuvre d'ethnologues? Quelle est la nature de leur engagement et à quels échanges peut-il donner lieu avec une discipline telle que l'anthropologie? Cet article montre que se jouent, notamment, dans la constitution d'un espace dialogique singulier, des questions aussi essentielles que celles qui touchent à la formation des conventions esthétiques et à leur nature socialement construite, à la portée sociale et politique du geste créatif, au rapport entre la pratique et la réflexivité. La question de l'appropriation de domaines théoriques par le monde de l'art ne se limite pas aux pratiques des artistes, mais se retrouve aussi dans le champ de la critique, au sein d'une revue d'art telle que Texte zur Kunst, laquelle a consacré un numéro entier à une notion telle que le réalisme spéculatif, en 2014. Les questions posées par Philippe Le Guern réapparaissent: $s$ 'agit-il d'un échange de bons procédés entre domaines séparés ou bien de pure appropriation de notions exogènes par le monde de l'art? S'agit-il d'un retour à la valorisation de l'expérience artistique et critique? Ce retour peut sembler paradoxal, car la philosophie spéculative tendrait plutôt à minimiser le rôle de l'appréhension humaine des phénomènes. L'expérience directe permise par la spéculation est en effet promue au détriment de la réflexion sur les conditions de cette expérience et de son écriture. 
Le dernier texte du dossier permet de revenir à des pratiques concrètes avec les interventions d'artistes autour d'objets coloniaux. Deux musées sont abordés par Marine Schütz: le Mucem de Marseille et le musée de Bristol, ce qui permet de constater leur ouverture, mais peut-être aussi leur volonté de mettre à profit - pour ne pas dire instrumentaliser - des artistes contemporains afin d'offrir au public l'image d'une plus grande ouverture aux questions qui agitent la société contemporaine. Nous publions également le témoignage de Lia Giraud, ancienne participante au programme SACRe, un programme qui met en relation des artistes contemporains et des centres de recherche autour d'un projet scientifique, lequel se concrétise par la réalisation d'un doctorat hybride. Ce témoignage permet d'envisager concrètement la richesse et les limites de ce genre de partenariat, tout en donnant un exemple concret de " circulation des idées dans les mondes de l'art».

Un entretien avec Laurent Jeanpierre, chercheur en sciences politiques - et simultanément acteur occasionnel du milieu de l'art - permet de donner un point de vue un peu différent sur les limites entre pratiques artistiques et théories issues des sciences humaines.

Pour le reste, le numéro consacre quelques pages à un portfolio de Yasmina Benabderrahmane et à des comptes rendus d'ouvrages et d'expositions.

Jérôme Glicenstein

Avril 2021 